Check for updates

Cite this: Mater. Horiz., 2020, 7, 1573

Received 3rd January 2020,

Accepted 26th March 2020

DOI: $10.1039 / \mathrm{d} 0 \mathrm{mh} 00014 \mathrm{k}$

rsc.li/materials-horizons

\section{Superfast and controllable microfluidic inking of anti-inflammatory melanin-like nanoparticles inspired by cephalopods $\dagger$}

\author{
Shiqi Wang, (D) $\ddagger^{\mathrm{a}}$ Saowanee Wannasarit, $\ddagger^{\mathrm{ab}}$ Patricia Figueiredo, ${ }^{a} \mathrm{Jiachen} \mathrm{Li},{ }^{\text {ac }}$ \\ Alexandra Correia, ${ }^{a}$ Bing Xia, (D) ${ }^{c}$ Ruedeekorn Wiwattanapatapee, ${ }^{b}$ Jouni Hirvonen, ${ }^{a}$ \\ Dongfei Liu, (D) ad Wei Li (D) *a and Hélder A. Santos (D) *ad
}

Here, a microfluidic approach for superfast melanin-like nanoparticle preparation with tunable size and monodispersity is reported. The particles formed have similar chemical composition to those prepared by the bulk method, and show reactive oxygen species scavenging behaviour and inflammatory macrophage phenotype switching capability, suggesting their potential for anti-inflammatory applications.

Cephalopods, including octopuses, cuttlefish and squids, are well-known for their unique inking behavior when disturbed. The dark-colored ink comprises melanin nanoparticles (MNs) secreted from the ink sac, as well as chemicals and polymeric contents from the funnel gland. ${ }^{1}$ Cephalopod ink has been used in medicine since ancient times both in Western and Eastern culture, without knowing the real active components. ${ }^{2}$ Recent studies found that MNs from Sepia ink had superior antioxidant potency due to the reactive oxygen species (ROS) scavenging capability. ${ }^{3-5}$ Considering the prevalent oxidative stress closely associated with inflammation, especially chronic non-resolving inflammation associated diseases, ${ }^{6,7}$ MNs from cephalopod ink may show promising potential in anti-inflammatory applications.

The biosynthesis of melanin in cephalopods is a highly regulated process confined in intracellular vesicles called melanosomes (0.5-1 $\mu \mathrm{m}$ in diameter), where tyrosine and 3,4-dihydroxyL-phenylalanine (DOPA) substrates undergo a series of enzymecascade reactions and finally form MNs. ${ }^{8}$ Unlike the ellipse-shaped

\footnotetext{
${ }^{a}$ Drug Research Program, Division of Pharmaceutical Chemistry and Technology, Faculty of Pharmacy, University of Helsinki, FI-O0014 Helsinki, Finland. E-mail:wei.li@helsinki.fi, helder.santos@helsinki.fi

${ }^{b}$ Department of Pharmaceutical Technology, Faculty of Pharmaceutical Sciences, Prince of Songkla University, 90110 Hat Yai, Thailand

${ }^{c}$ Key Laboratory of Forest Genetics \& Biotechnology (Ministry of Education of China), College of Science, Nanjing Forestry University, 210037 Nanjing, P. R. China

${ }^{d}$ Helsinki Institute of Life Science (HiLIFE), University of Helsinki, FI-00014 Helsinki, Finland

$\dagger$ Electronic supplementary information (ESI) available: Materials, experimental details and additional data. See DOI: 10.1039/d0mh00014k

\$ These authors contributed equally to this work.
}

\begin{abstract}
New concepts
We propose a new concept utilizing the microfluidic technique for superfast, controllable and monodisperse melanin nanoparticle (MN) production, inspired by MN biosynthesis from cephalopods. With high biocompatibility, biodegradability and free radical scavenging abilities, melanin has been intensively used as an antioxidant, photothermal agent and surface coating material in the biomedical field. Although traditional MN preparation methods generate homogenous nanoparticles, the lengthy reaction times and unavoidable batch-to-batch variations limit the possibility to tailor particle production towards real-world applications. Thanks to the microfluidic technique, here we demonstrate that monodisperse MNs can be produced in a highly concentrated substrate solution within seconds for the first time. By simply adjusting the parameters of the microfluidic device, we can precisely manipulate the size and the morphology of the produced nanoparticles. These MNs made with the microfluidic method showed similar chemical composition to those prepared by the bulk method. The negligible toxicity towards macrophages, reactive oxygen species scavenging capability, and inflammation attenuating effects towards M1 phenotype macrophages suggest their potential for anti-inflammatory applications.
\end{abstract}

melanin microparticles produced by vertebrates, MNs produced by cephalopods are uniform and spherical, with an average size of approximately $150 \mathrm{~nm} .{ }^{9}$ By transmission electron microscopy (TEM) imaging, it was identified that one melanosome can accommodate up to 30 MNs. ${ }^{10}$ Such concentrated MNs made in the melanosomes are speculated to be synthesized from locally concentrated substrates in a highly catalytic environment, somehow uniquely developed in evolution.

Inspired by the efficient and well-controlled biosynthesis of MNs, we developed a glass capillary-based microfluidic device for producing monodisperse MNs from concentrated dopamine with fine-tuning of the size and morphology (Fig. 1a). Although some artificial MN preparation methods have been reported by bottom-up self-polymerization in alkaline solutions, ${ }^{11-13}$ only very dilute substrates were allowed at slow reaction rates (typically 1-3 day reaction time) to prepare homogenous MNs. At a higher substrate concentration, the reaction becomes too fast, leading to uncontrollable nanoparticle (NP) formation with heterogeneous properties. In contrast, microfluidic technology enables precise 


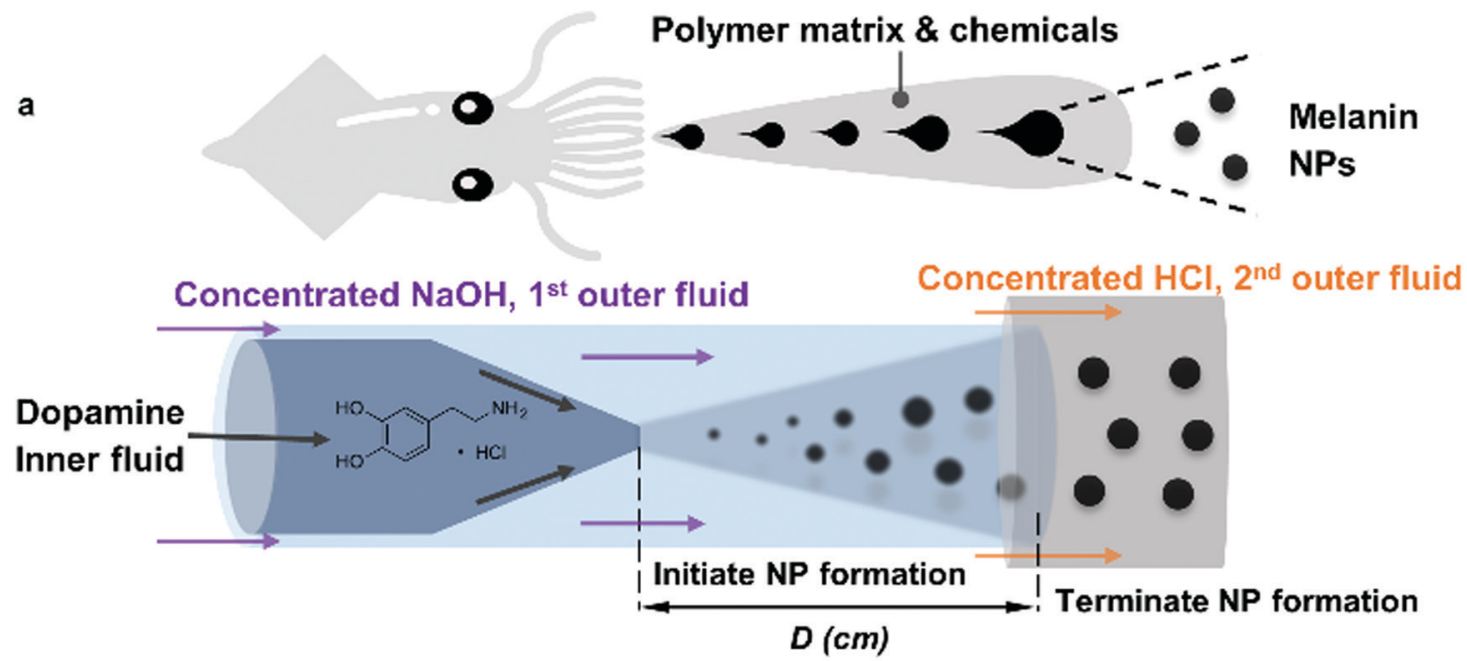



e

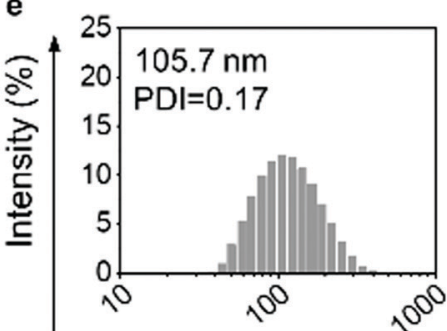

$D=4.5$



f



$D=12$

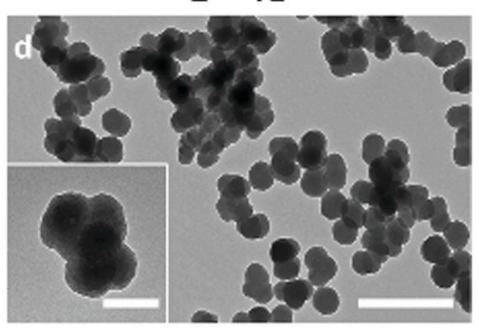

g

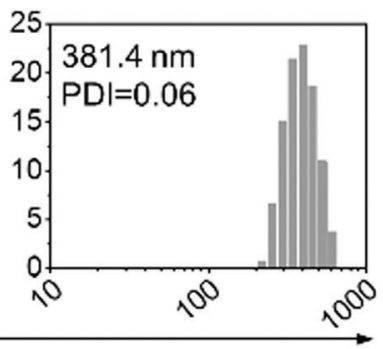

Size $(\mathrm{nm})$

Fig. 1 (a) The schematic illustration of MN preparation on a co-flow glass-capillary microfluidic device in parallel with a cuttlefish inking comparison. $(b-d)$ TEM images of the MNs prepared by the microfluidic device with different parameters $(D=3,4.5$ and $12 \mathrm{~cm}$, respectively). Scale bar $=500 \mathrm{~nm}$ in the main image and $100 \mathrm{~nm}$ in the inset. (e-g) DLS size distribution profiles of the MNs prepared by the microfluidic device with different parameters $(D=3,4.5$ and $12 \mathrm{~cm}$, respectively).

control of superfast liquid mixing and mass transfer in complex, miniaturized capillary networks. ${ }^{14-20}$ Within milliseconds, all the reagents efficiently mix, resulting in a homogenous environment throughout. $^{21-23}$

As shown in Fig. 1a, the co-flow microfluidic device for MN production is composed of three sequentially aligned glass capillaries. Dopamine hydrochloride was dissolved in Milli-Q water as the inner fluid and pumped into the inner tapered glass capillary, while concentrated sodium hydroxide $(\mathrm{NaOH})$ solution, as the 1st outer fluid, was pumped between the inner and 1st outer cylindrical capillary. Once mixed with $\mathrm{NaOH}$, dopamine started self-polymerization followed by oligomer aggregation to form MNs. Then, the 2nd outer fluid, concentrated hydrochloric acid $(\mathrm{HCl})$, was pumped into the space between the 1 st and the 2nd outer cylindrical capillaries. When the MNs in the 1st outer fluid encountered the $\mathrm{HCl}$, the self-polymerization of dopamine, which is $\mathrm{pH}$-dependent, was terminated due to the acidification of the solutions. Thus, by controlling the distance (marked as $D$ in Fig. 1a) between the tip of the tapered capillary and the end of the 1st outer cylindrical capillary, the reaction time of dopamine polymerization was precisely controlled, which in turn tuned the size and morphology of the MNs. The variation of $D$ shows the time resolution of $\mathrm{MN}$ particle formation by spatial resolution within the microfluidic chip, which provides unique insight into the reaction kinetics.

The size and morphology of the MNs produced by the microfluidic device with different $D$ values $(3 \mathrm{~cm}, 4.5 \mathrm{~cm}$ and $12 \mathrm{~cm}$ ) were characterized by dynamic light scattering (DLS) and TEM. As shown in Fig. 1b-d, when $D$ increased from 3 to $12 \mathrm{~cm}$, the size of the MNs increased and the particles became 
denser with higher contrast in TEM images. The DLS results also confirm the trend, because the whole distribution profile shifted to larger sizes with an increase in $D$ (Fig. 1e-g). Within $5.08 \mathrm{~s}(D=3 \mathrm{~cm})$, loosely assembled NPs with slightly irregular shape (Fig. 1b and inset) were already formed. The rough surface was possibly due to the random aggregation in such a short time. The particle size shown in TEM was smaller than that measured by DLS (Fig. 1e), suggesting that the NPs were possibly quite swollen and hydrated. ${ }^{13}$ After another $2.54 \mathrm{~s}$ $(D=4.5 \mathrm{~cm})$, the hydrodynamic size of the NPs increased by $31 \mathrm{~nm}$, with a smaller PDI. Notably, the MNs became rounder, solid and smooth (Fig. 1c and inset), indicating that the particle growth homogenized the particle surface, and the size distribution became narrower. On elongating the reaction time to $20.32 \mathrm{~s}$ ( $D=12 \mathrm{~cm}$ ), the hydrodynamic size of the MNs increased to $381 \mathrm{~nm}$, but still with monodispersity (Fig. 1d and g). At this stage, the aggregation became more evident, and more merged particles could be identified in the TEM image. The overall results shown above suggest that monodisperse MNs of controllable sizes can be easily prepared within a few seconds by this novel microfluidic device, using 100 times more concentrated dopamine substrate (200 $\mathrm{mg} \mathrm{mL}^{-1}$ dopamine) compared with the conventional bulk method. $^{13}$

In comparison, we also used the same condition to produce MNs by the conventional bulk method. After rapidly mixing dopamine and $\mathrm{NaOH}$ solutions, $\mathrm{HCl}$ was immediately added to terminate the reaction. However, the MNs synthesized were polydisperse with micro-size aggregates (Fig. 2a and b). This was possibly because the reaction and subsequent particle formation were faster than the mixing. Therefore, the spatial and temporal heterogeneities led to uncontrollable particle formation and aggregation. A further increase of the reaction time to $20 \mathrm{~h}$ did not make the particles small or homogenous (Fig. S1, ESI $\dagger$ ), suggesting that the aggregation right after the $\mathrm{NaOH}$ addition was irreversible.

Then, we decreased the dopamine concentration to $1.5 \mathrm{mg} \mathrm{mL}^{-1}$, close to the concentration range reported in conventional bulk $\mathrm{MN}$ synthesis methods. ${ }^{13}$ As shown in Fig. 2c, MN formation was significantly slower at such a low concentration of dopamine. Although the colour of the reaction solution changed to dark yellow immediately after the reaction was initiated (Fig. S2, $\mathrm{ESI} \dagger)$, the count rate in the first $5 \mathrm{~min}$ remained almost the same, without any identifiable particles (Fig. S3, ESI $\dagger$ ). After 5 min, the count rate started to increase, indicating the formation of nanoparticles with light-scattering capabilities. During the first $1 \mathrm{~h}$, the $Z$-average size of the particles remained almost the same, with the PDI greatly decreased. At the same time, the count rate increased. Since the count rate is proportional to $c \times r^{6}(c$ represents the particle concentration and $r$ represents the particle size) ${ }^{24}$ this means that the number of MNs in the solution increased during the first hour, probably due to the particle nucleation. The supersaturation of soluble oligomers caused MNs of around $30 \mathrm{~nm}$ to nucleate continuously during this initial stage. Afterwards, the particle size grew steadily with time, with a slightly increased PDI, suggesting slow particle growth. After $24 \mathrm{~h}$, the particles reached a size of $65.3 \mathrm{~nm}$ with a PDI of 0.16 (Fig. S4, ESI $\dagger$ ). A further increase in the reaction time led to larger aggregates $(104.2 \mathrm{~nm}$ at $48 \mathrm{~h}$ and $262.3 \mathrm{~nm}$ at $72 \mathrm{~h}$ ) with larger size distribution.

Given the above results, we made a comparison of the MN formation kinetics with the bulk method using diluted dopamine

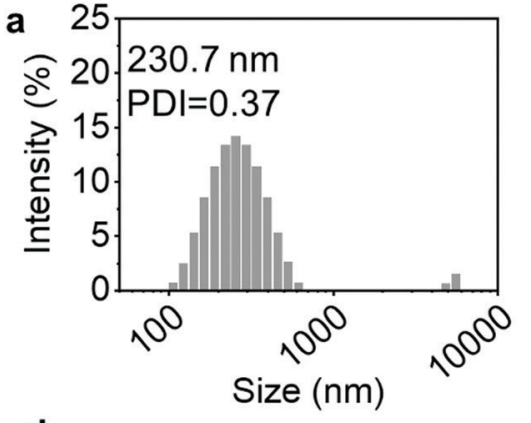

d

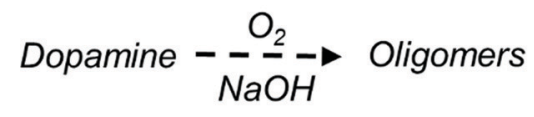
$<5 \min$



$\stackrel{\text { Nucleation }}{\rightarrow}$ Seeds

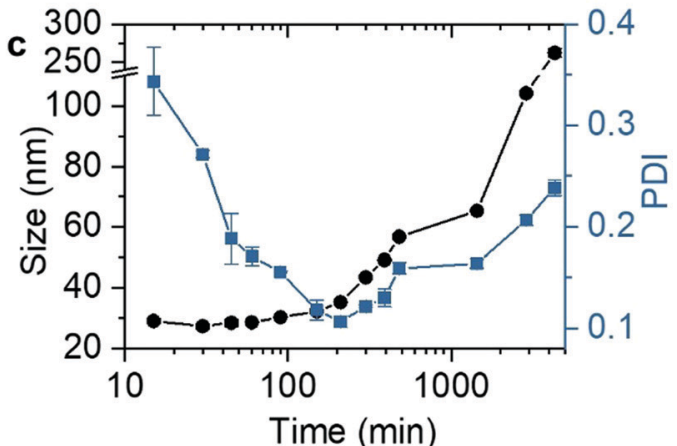

Time (min)

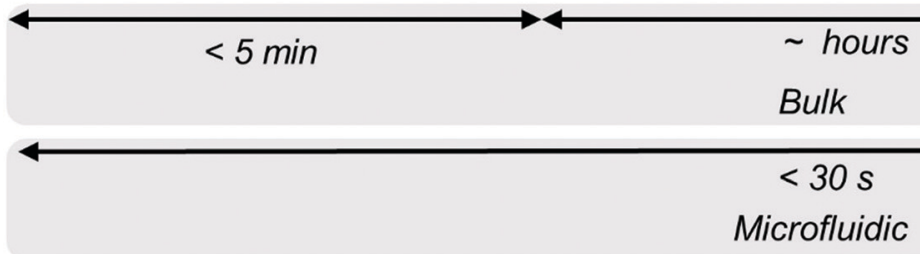

Fig. 2 (a and b) DLS distribution profile and TEM image of the MNs prepared by the bulk method at $200 \mathrm{mg} \mathrm{mL}^{-1}$ dopamine. Scale bar $=500 \mathrm{~nm}$. (c) The $Z$-average size and PDI of MNs prepared by the bulk method at $1.5 \mathrm{mg} \mathrm{mL}^{-1}$ dopamine. (d) The schematic showing MN formation kinetic steps in the conventional bulk method and microfluidic method. 
and with the microfluidic method using concentrated dopamine. According to the literature, the generally accepted simplified MN formation mechanism involves 4 steps, including dopamine oxidation, oligomer formation, oligomer aggregation, and aggregate growth (Fig. 2d). ${ }^{25}$ First, dopamine was auto-oxidized by the dissolved oxygen in the alkaline solution to form quinone, and further converted to 5,6-dihydroxyindole (DHI) and indole-5,6quinone (IDQ) ${ }^{26}$ Further oxidation of DHI and IDQ leads to the formation of oligomers (1.0-1.5 nm), which quickly aggregate and form "seeds". 27 The polymer grows steadily from these "seeds" by continuous addition of monomers or dimers by covalent or non-covalent bonding during the polymerization process, ${ }^{28,29}$ finally generating nano- or micro-sized particles.

At a low concentration of dopamine, the oxidation and oligomer formation were slow ( $>5 \mathrm{~min}$ ). This allows for thorough mixing of all reactants in the solution before reaching the supersaturation of MN precursors. Therefore, the nucleation proceeded in a relatively homogenous environment, and the subsequent growth in such a dilute solution was possibly diffusion-controlled, resulting in monodisperse particles after hours or days. In the microfluidic system, all the steps were superfast, due to the high concentration of dopamine. The mixing was also fast and highly efficient, leading to a similarly homogenous environment for nanoparticle nucleation. Therefore, monodisperse particles, with precisely controlled initiation of self-polymerization of dopamine, aggregation of oligomers and termination of the reaction, can be produced in a microfluidic device within a few seconds.

As the MN formation kinetics on microfluidic chips differed from that of conventional bulk methods, we hypothesized that the structure and physiochemical properties were affected by the preparation methods. We first compared the size and morphology of the synthetic MNs with natural MNs from Sepia, which is considered as a standard of eumelanin. ${ }^{30}$ The Sepia MNs acquired from a commercial supplier after reconstitution in water showed a broad distribution profile with more than one peak (Fig. S5, ESI $\dagger$ ). Even after the removal of aggregates using low speed centrifugation, there were still some microsized particles, as shown in the DLS size distribution profile (Fig. 3a). The morphology of these particles was compact and spherical (Fig. 3b), consistent with previous literature. ${ }^{13}$ The zeta-potential of all MNs was similar (around $-30 \mathrm{mV}$ ) (Table $\mathrm{S} 1$, ESI $\dagger$ ). Further stability studies at $37{ }^{\circ} \mathrm{C}$ in $50 \%$ human plasma showed no obvious size change over a $2 \mathrm{~h}$ incubation period (Fig. S6, ESI $\dagger$ ). The PDI of the MNs in plasma was higher than that in water, probably due to the high protein content in the plasma, which caused a bimodal distribution. ${ }^{31,32}$

The chemical composition of MNs prepared by the microfluidic technique, prepared by the bulk method (slow reaction) and natural MNs from Sepia was examined by Fourier-transform infrared spectroscopy (FTIR). As shown in Fig. 3c, the MNs from Sepia had broad bands around 3380, 1590 and $1354 \mathrm{~cm}^{-1}$, corresponding to $\mathrm{N}-\mathrm{H}$ stretching from secondary amine, $\mathrm{C}=\mathrm{C}$ aromatic ring stretching and $\mathrm{C}-\mathrm{N}-\mathrm{C}$ stretching from indole rings. ${ }^{33}$ The broad bands clearly differed from those sharp and split bands shown in the dopamine FTIR spectrum, indicating the heterogeneous polymeric nature of the MNs. The frequencies and the band shape are in good agreement with published frequencies of similar samples. ${ }^{33,34}$
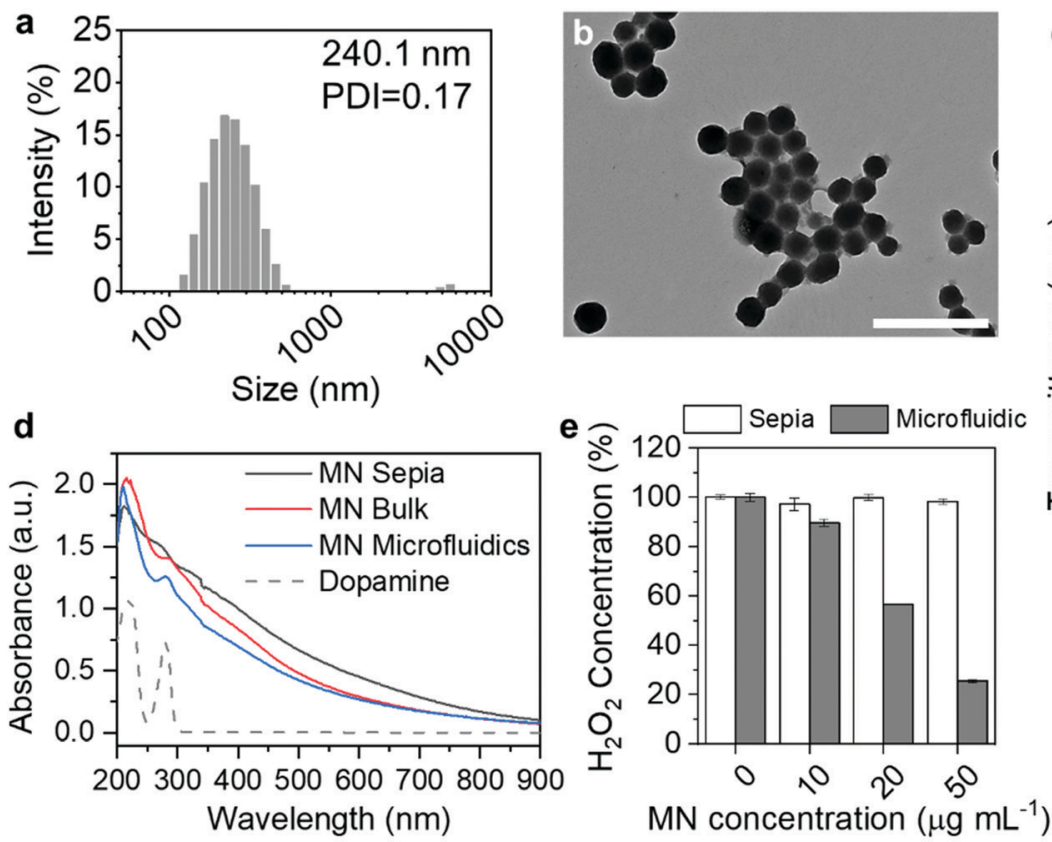

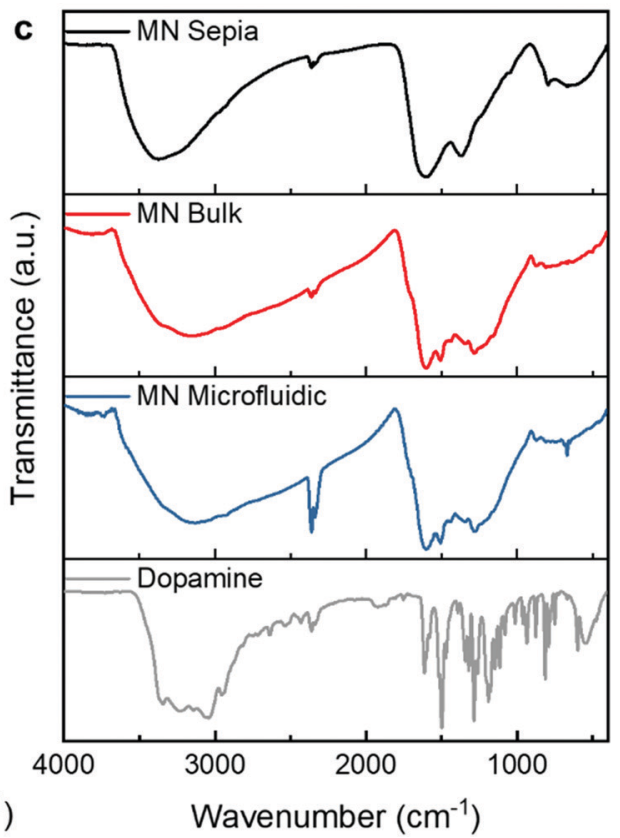

Fig. 3 (a and b) DLS distribution profile and TEM image of the MNs from Sepia after low speed centrifugation. Scale bar $=500 \mathrm{~nm}$. (c) The FTIR spectra of MNs from Sepia (black), MNs synthesized by the bulk method (red), MNs synthesized by the microfluidic method (blue) and dopamine (grey). (d) The UV-vis absorbance spectra of MNs from Sepia (black), MNs synthesized by the bulk method (red), MNs synthesized by the microfluidic method (blue) and dopamine (grey dash line). (e) The $\mathrm{H}_{2} \mathrm{O}_{2}$ scavenging capability of $\mathrm{MNs}$ at different concentrations $\left(10,20\right.$ and $50 \mu \mathrm{g} \mathrm{mL}^{-1}$ ). The results were normalized to the untreated controls. 
The synthetic MNs showed identical FTIR spectra, regardless of the preparation method (Fig. 3c). Notably, the synthetic MNs' spectra showed several different bands between 1800 and $900 \mathrm{~cm}^{-1}$ compared with the natural MNs. First, there is an additional band at $1715 \mathrm{~cm}^{-1}$, corresponding to $\mathrm{C}=\mathrm{O}$ stretching (zoomed in FTIR spectra in Fig. S7, ESI $\dagger$ ). This carbonyl group may originate from the ketonic carbonyl of quinone groups, or from the carboxylic acids on dihydroxyindole-2carboxylic acid (DHICA) and other derivatives. ${ }^{35}$ Other differences include a series of bands emerging at 1610, 1510 and $1436 \mathrm{~cm}^{-1}$, compared with one broad and smooth band in the natural MNs' spectrum. The multiple bands are all correlated to the $\mathrm{C}=\mathrm{C}$ aromatic ring stretching in different chemical environments, suggesting a heterogeneous structure. The strong bands at 1280 and $1224 \mathrm{~cm}^{-1}$ corresponding to the $\mathrm{C}-\mathrm{O}$ vibration might originate from the hydroxyl groups from $\mathrm{DHI} .{ }^{33,34}$ These differences in the FTIR spectra suggest that the chemical composition of synthetic MNs from the bulk and microfluidic methods is similar, but differs from the natural MNs.

The UV absorbance spectra of all MNs (Fig. 3d) showed typical broad absorbance from UV to near infra-red wavelengths, due to the chemical and structural disorder. ${ }^{36,37}$ The shapes of the absorbance spectra of MNs prepared by the microfluidic and MNs prepared by the bulk method are identical, with a small peak at $280 \mathrm{~nm}$, suggesting residual dopamine or other low molecular weight precursors absorbed on the nanoparticles. MNs from Sepia had a smoother absorbance profile without any obvious peak, and the absorbance is slightly higher than that of the synthetic MNs in the infra-red region, which suggests that there might be some differences in the hierarchical assembly structure within the particles. $^{38}$

Next, we evaluated the ROS scavenging capability of MNs synthesized by the microfluidic method. We first evaluated whether the MNs can scavenge hydrogen peroxide $\left(\mathrm{H}_{2} \mathrm{O}_{2}\right)$, which is a main type of cellular ROS. ${ }^{7,39}$ MNs were incubated with $\mathrm{H}_{2} \mathrm{O}_{2}(10 \mu \mathrm{M}$, which is detrimental to cells and leads to necrosis ${ }^{40,41}$ ) for $1 \mathrm{~h}$ and the remaining $\mathrm{H}_{2} \mathrm{O}_{2}$ concentration was evaluated by Amplex ${ }^{\mathrm{TM}}$ UltraRed reagent. As shown in Fig. 3e, the $\mathrm{H}_{2} \mathrm{O}_{2}$ scavenging capability of the MNs is concentration-dependent. At $20 \mu \mathrm{g} \mathrm{mL}{ }^{-1}$, the MNs decreased the $\mathrm{H}_{2} \mathrm{O}_{2}$ concentration by half, and further decreased it to $25 \%$ at a concentration of $50 \mu \mathrm{g} \mathrm{mL} \mathrm{m}^{-1}$. The $\mathrm{H}_{2} \mathrm{O}_{2}$ scavenging activity might be attributed to the DHICA moieties in the MNs, as shown in the FTIR spectrum.

Surprisingly, the Sepia MNs did not show $\mathrm{H}_{2} \mathrm{O}_{2}$ scavenging capability in the studied concentration range (Fig. 3e). It has long been known that Sepia MNs react with $\mathrm{H}_{2} \mathrm{O}_{2},{ }^{42}$ and a high concentration of $\mathrm{H}_{2} \mathrm{O}_{2}$ bleaches natural MNs generated by various species. ${ }^{43}$ However, most previous studies regarding Sepia MNs used fresh MNs purified from Sepia ink, instead of lyophilized samples from a commercial supplier. There is evidence showing that the purification method affected the composition of the final $\mathrm{MNs},{ }^{44}$ as well as the morphology. ${ }^{9}$ It is likely that the commercial MNs, after purification, lyophilization and rehydration, lost the $\mathrm{H}_{2} \mathrm{O}_{2}$ scavenging capability.

Based on the $\mathrm{H}_{2} \mathrm{O}_{2}$ scavenging capability of the MNs prepared by the microfluidic technique, we decided to investigate the antioxidant and anti-inflammatory potential of the material in biological systems. As a proof-of-concept study, we decided to evaluate the MNs on macrophage cells, which are key players in inflammation development. Pro-inflammatory macrophages (M1 phenotype) secrete high levels of pro-inflammatory cytokines such as tumor necrosis factor-alpha (TNF- $\alpha$ ), interleukin (IL)-1 $\beta$, IL-12 and IL-23, and contribute to inflammation progression. ${ }^{45-47}$ In contrast, the M2 phenotype secretes anti-inflammatory cytokines (e.g., IL-10 and transforming growth factor-beta) and promotes tissue repair. ${ }^{48-50}$ Therefore, switching the M1 to the M2 phenotype using anti-inflammatory nanoparticles is a promising strategy for anti-inflammatory therapy. ${ }^{51}$

Before the evaluation of the anti-inflammatory potency, we first tested the cytotoxicity of the MNs on RAW 264.7 cells, which are typical murine macrophages. The cells were cultured with MNs for 6, 24 and $48 \mathrm{~h}$, respectively, and the cell viability was assessed by the CellTiter-Glo ${ }^{\mathbb{R}}$ luminescence assay. As shown in Fig. 4a, the MNs had negligible cytotoxicity at concentrations lower than $100 \mu \mathrm{g} \mathrm{mL} \mathrm{m}^{-1}$. When the concentration increased to $500 \mu \mathrm{g} \mathrm{mL} \mathrm{m}^{-1}$, the cell viability was $75 \%$ at $6 \mathrm{~h}, 68 \%$ at $24 \mathrm{~h}$ and
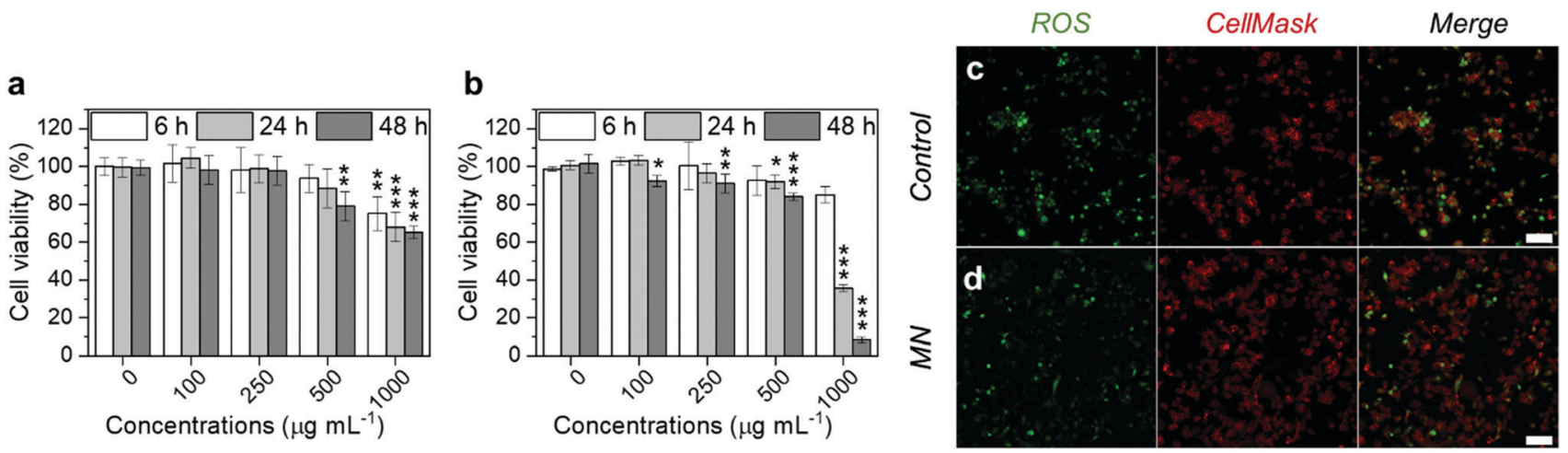

Fig. 4 (a and b) The cytotoxicity of MNs prepared by the microfluidic method on RAW 264.7 cells (a) and THP-1 cells (b) after 6, 24 and $48 \mathrm{~h}$ incubation. The results were normalized to the untreated controls. Data are presented as the mean \pm s.d. $(n=4)$. ${ }^{*} P<0.05, * * P<0.01$, and ${ }^{* \star *} P<0.001$. (c and d) The confocal images showing the ROS level in RAW 264.7 cells stimulated by LPS and IFN- $\gamma$ for $24 \mathrm{~h}$, and then treated without particles (c) and with MNs at $20 \mu \mathrm{g} \mathrm{mL} \mathrm{m}^{-1}$ (d). The intracellular ROS level was indicated by the green fluorescence emitted by dichlorofluorescein. The plasma membranes were stained by CellMask ${ }^{\mathrm{TM}}$ Deep Red to outline the cells. 
$65 \%$ at $48 \mathrm{~h}$. The increased cytotoxicity was probably due to the cellular uptake of MNs, which was confirmed by flow cytometry and confocal microscopy (Fig. S8, ESI $\dagger$ ). We further tested the cytotoxicity on THP-1 cells (human monocytes, which are commonly used for macrophage differentiation). The results were similar to those of RAW 264.7 (Fig. 4b), which showed decreased cell viability when the concentration was higher than $100 \mu \mathrm{g} \mathrm{mL}^{-1}$.

The ROS scavenging capability of the MNs was evaluated on inflamed RAW 264.7 cells. Prior to the NP incubation, RAW 264.7 cells were stimulated by lipopolysaccharides (LPS) and interferon-gamma (IFN- $\gamma$ ), which are common reagents for inducing oxidative stress and inflammatory responses. ${ }^{46}$ Then the cells were treated with MNs for $5 \mathrm{~h}$, before analyzing using the oxidant-sensing fluorescent probe $2^{\prime}, 7^{\prime}$-dichlorodihydrofluorescein diacetate (DCFH-DA). Non-polar DCFH-DA can diffuse through cell membranes into the cytoplasm where it gets hydrolyzed by esterases and subsequently oxidized by ROS to become fluorescent. ${ }^{52}$ The fluorescence intensity of all cells was then analyzed by confocal microscopy. As shown in Fig. $4 \mathrm{c}$ and $\mathrm{d}$, the prevalent green fluorescence emitted by oxidised DCFH in the positive control (cells stimulated by LPS and IFN- $\gamma$ ) confirmed the high ROS level. Cells treated with MNs showed slightly decreased green fluorescence compared with the positive control, although some cells were not fully recovered, possibly due to the short incubation time.

Further characterization of the ROS scavenging capability was performed on RAW 264.7 cells challenged by concentrated $\mathrm{H}_{2} \mathrm{O}_{2}$. Quantitative analysis using DCFH-DA suggested that the cells treated with MNs showed a lower ROS level compared with the positive control, and a higher concentration of MNs led to better scavenging effects (Fig. S9, ESI $\dagger$ ). At $50 \mu \mathrm{g} \mathrm{mL}{ }^{-1}$ of MNs, the ROS level became $10 \%$ lower after 45 min challenge, and
$20 \%$ lower when the challenge time was elongated to $90 \mathrm{~min}$. We also used a small molecule ROS scavenger, L-glutathione (GSH), as a control. The preloaded GSH caused a $14 \%$ decrease in ROS at $45 \mathrm{~min}$, but almost no effects at $90 \mathrm{~min}$, probably due to the fast metabolism. ${ }^{53}$

Next, we evaluated the anti-inflammatory therapeutic efficacy on RAW 264.7 macrophages. As shown in Fig. 5a, the macrophages in the resting state (M0) were first stimulated by LPS and IFN- $\gamma$ for 1 day, and then treated with MNs. The cells stimulated by LPS and IFN- $\gamma$ but without any treatment developed into the M1 phenotype (M1 control), while those M0 cells with antiinflammatory cytokine stimulation (IL-4 and IL-13) for 3 consecutive days developed into the M2 phenotype (M2 control). The M1 and M2 phenotypes have distinguishable markers on the surface and cytokine secretion profiles. ${ }^{46}$ Therefore, we evaluated the expression of CD80 (M1 marker) and CD206 (M2 marker) by immunostaining and subsequent analysis by flow cytometry, as well as the concentration of TNF- $\alpha$ (M1 cytokines) and IL-10 (M2 cytokines) by enzyme-linked immunosorbent assay (ELISA), to identify the phenotypes after treatment.

As shown in Fig. $5 \mathrm{~b}$ and c, MNs reduced the pro-inflammatory cytokine TNF- $\alpha$ effectively to almost the Mo level. At the same time, the anti-inflammatory cytokine IL-10 secretion was elevated, suggesting potent inflammation-attenuating effects. Further investigation on the cell marker by flow cytometry showed that the M1 typical marker CD80 of MN treated cells decreased significantly compared with the M1 control (Fig. 5d). The M1 phenotype population $\left(\mathrm{CD}^{+} 0^{+} \mathrm{CD} 206^{-}\right)$decreased from $92.2 \%$ to $27.3 \%$, with an increase of $10 \%$ in the M2 phenotype (CD80 $\mathrm{CD}_{206}{ }^{+}$) population. Around half of the cells (52.1\%) became double negative, suggesting the transformation into a resting
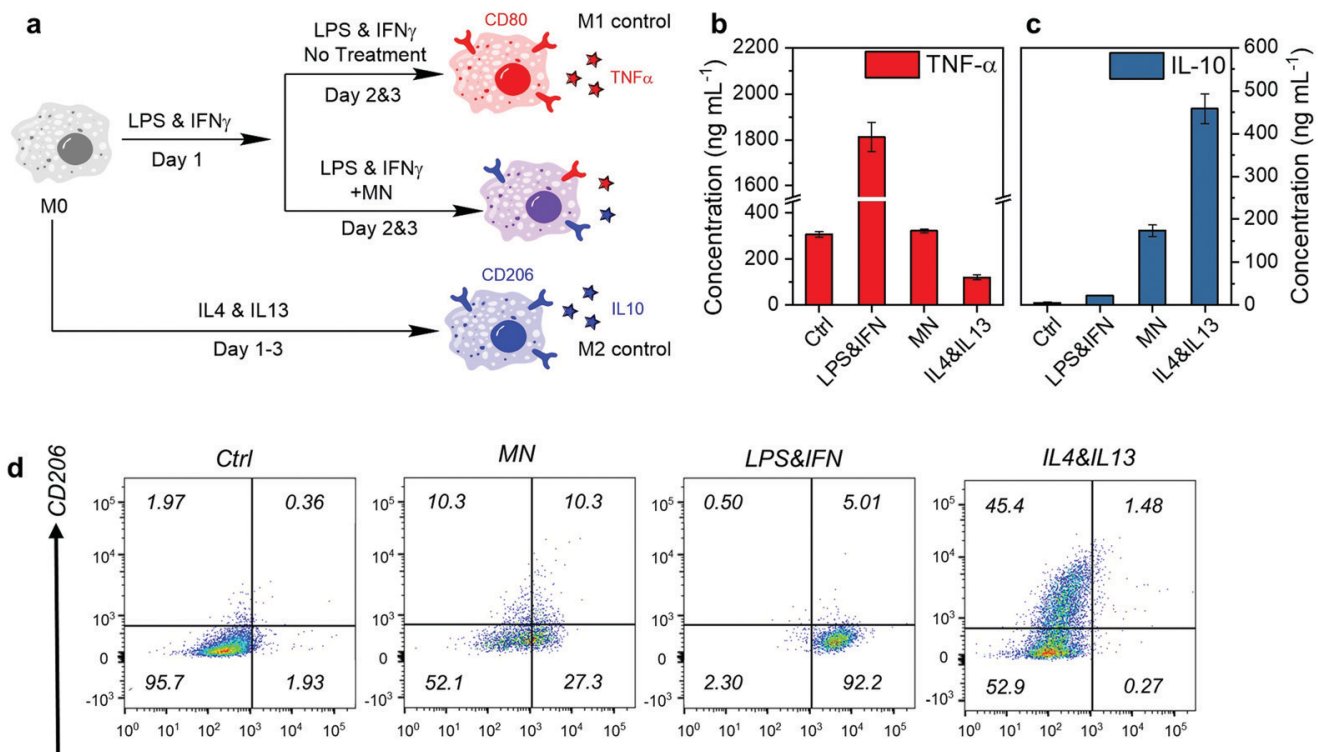

Fig. 5 (a) Schematic representation of the macrophage stimulation and treatment. (b and c) The concentration of TNF- $\alpha$ and IL-10 in the macrophage culture medium after stimulation and treatment, quantified by ELISA. Data are presented as the mean \pm s.d. ( $n=3$ ). (d) The flow cytometry analysis of macrophage marker CD80 and CD206 expression after immunostaining. Control (ctrl) represents cells without LPS and IFN- $\gamma$ stimulation. MN represents cells stimulated by LPS and IFN- $\gamma$ and treated with MNs at $20 \mu \mathrm{g} \mathrm{mL}^{-1}$ for 2 days. LPSEIFN represents the M1 control with continuous pro-inflammatory stimulation, while IL4\&IL13 represents the M2 control with continuous anti-inflammatory stimulation. 
M0 state. This means that the inflammation attenuation effect of the MNs was due to the macrophage phenotype conversion. The molecular mechanism behind the phenotype conversion by MNs is possibly related to the down-regulation of Toll-like receptor 4 expression (for sensing LPS) and NF-אB signaling pathway. ${ }^{54}$

\section{Conclusions}

In this study, we developed melanin-like nanoparticles by superfast and controlled preparation in microfluidic devices inspired by cephalopods. The MNs were generated from highly concentrated substrates within seconds, which is comparable to the biosynthesis. Characterized by DLS and TEM, the particles showed spherical morphology and monodisperse size, due to the superfast mixing and the precise control of initiation and termination of the reaction. Furthermore, the MNs prepared by the microfluidic method successfully reduced the pro-inflammatory cytokine level and induced the secretion of immune-suppressive cytokines IL-10 on macrophages, and altered the cell phenotype. The simple but advanced preparation of MNs by a microfluidic device, along with the inflammation attenuating property, makes the nanoparticles developed here a promising candidate for antiinflammatory applications.

\section{Experimental}

Details of the experimental procedures are provided in the ESI. $\dagger$

\section{Conflicts of interest}

The authors declare no conflicts of interest.

\section{Acknowledgements}

Dr S. Wang acknowledges the financial support from the Jenny and Antti Wihuri Foundation. S. Wannasarit acknowledges financial support from the Thailand Research Fund under the Royal Golden Jubilee (RGJ) PhD program (PHD/0180/2556). Dr W. Li acknowledges financial support from the Finnish Cultural Foundation (grant no. 00190634) and the Academy of Finland (decision no. 322093). Prof. H. A. Santos acknowledges financial support from the HiLIFE Research Funds, the Sigrid Jusélius Foundation, the Norwegian Research Council/Nacamed AS, and the European Research Council Proof-of-Concept Research Grant (grant no. 825020). The authors also acknowledge the following core facilities funded by Biocenter Finland: Electron Microscopy Unit for TEM, the Light Microscopy Unit of the Institute of Biotechnology for the confocal microscope and the Flow Cytometry Unit for the flow cytometry analyser.

\section{Notes and references}

1 C. D. Derby, Biol. Bull., 2007, 213, 274-289.

2 C. Derby, Mar. Drugs, 2014, 12, 2700-2730.

3 B.-L. L. Seagle, E. M. Gasyna, W. F. Mieler and J. R. Norris, Proc. Natl. Acad. Sci. U. S. A., 2006, 103, 16644-16648.
4 J.-P. Zhong, G. Wang, J.-H. Shang, J.-Q. Pan, K. Li, Y. Huang and H.-Z. Liu, Mar. Drugs, 2009, 7, 9-18.

5 X. Guo, S. Chen, Y. Hu, G. Li, N. Liao, X. Ye, D. Liu and C. Xue, J. Food Sci. Technol., 2014, 51, 3680-3690.

6 S. K. Biswas, Oxid. Med. Cell. Longevity, 2016, 2016, 1-9.

7 M. Mittal, M. R. Siddiqui, K. Tran, S. P. Reddy and A. B. Malik, Antioxid. Redox Signaling, 2014, 20, 1126-1167.

8 A. Palumbo, Pigm. Cell Res., 2003, 16, 517-522.

9 Y. Liu and J. D. Simon, Pigm. Cell Res., 2003, 16, 72-80.

10 U. Schraermeyer, Pigm. Cell Res., 1994, 7, 52-60.

11 K. Ai, Y. Liu, C. Ruan, L. Lu and G. M. Lu, Adv. Mater., 2013, 25, 998-1003.

12 J. Yan, L. Yang, M.-F. Lin, J. Ma, X. Lu and P. S. Lee, Small, 2013, 9, 596-603.

13 K.-Y. Ju, Y. Lee, S. Lee, S. B. Park and J.-K. Lee, Biomacromolecules, 2011, 12, 625-632.

14 D. Liu, H. Zhang, F. Fontana, J. T. Hirvonen and H. A. Santos, Adv. Drug Delivery Rev., 2018, 128, 54-83.

15 D. Liu, S. Cito, Y. Zhang, C.-F. Wang, T. M. Sikanen and H. A. Santos, Adv. Mater., 2015, 27, 2298-2304.

16 D. Liu, H. Zhang, S. Cito, J. Fan, E. Mäkilä, J. Salonen, J. Hirvonen, T. M. Sikanen, D. A. Weitz and H. A. Santos, Nano Lett., 2017, 17, 606-614.

17 W. Li, D. Liu, H. Zhang, A. Correia, E. Mäkilä, J. Salonen, J. Hirvonen and H. A. Santos, Acta Biomater., 2017, 48, 238-246.

18 F. Araújo, N. Shrestha, M.-A. Shahbazi, D. Liu, B. HerranzBlanco, E. M. Mäkilä, J. J. Salonen, J. T. Hirvonen, P. L. Granja, B. Sarmento and H. A. Santos, ACS Nano, 2015, 9, 8291-8302.

19 H. Zhang, W. Cui, X. Qu, H. Wu, L. Qu, X. Zhang, E. Mäkilä, J. Salonen, Y. Zhu, Z. Yang, D. Chen, H. A. Santos, M. Hai and D. A. Weitz, Proc. Natl. Acad. Sci. U. S. A., 2019, 116, 7744-7749.

20 D. Liu, H. Zhang, F. Fontana, J. T. Hirvonen and H. A. Santos, Lab Chip, 2017, 17, 1856-1883.

21 R. Karnik, F. Gu, P. Basto, C. Cannizzaro, L. Dean, W. KyeiManu, R. Langer and O. C. Farokhzad, Nano Lett., 2008, 8, 2906-2912.

22 Y. Song, J. Hormes and C. S. S. R. Kumar, Small, 2008, 4, 698-711.

23 H. Song and R. F. Ismagilov, J. Am. Chem. Soc., 2003, 125, 14613-14619.

24 J. Shang and X. Gao, Chem. Soc. Rev., 2014, 43, 7267-7278. 25 M. Arzillo, G. Mangiapia, A. Pezzella, R. K. Heenan, A. Radulescu, L. Paduano and M. D'Ischia, Biomacromolecules, 2012, 13, 2379-2390.

26 N. F. Della Vecchia, R. Avolio, M. Alfè, M. E. Errico, A. Napolitano and M. D'Ischia, Adv. Funct. Mater., 2013, 23, 1331-1340.

27 N. F. Della Vecchia, A. Luchini, A. Napolitano, G. D’Errico, G. Vitiello, N. Szekely, M. D'Ischia and L. Paduano, Langmuir, 2014, 30, 9811-9818.

28 S. Hong, Y. S. Na, S. Choi, I. T. Song, W. Y. Kim and H. Lee, Adv. Funct. Mater., 2012, 22, 4711-4717.

29 D. R. Dreyer, D. J. Miller, B. D. Freeman, D. R. Paul and C. W. Bielawski, Langmuir, 2012, 28, 6428-6435. 
30 M. d'Ischia, K. Wakamatsu, A. Napolitano, S. Briganti, J.-C. Garcia-Borron, D. Kovacs, P. Meredith, A. Pezzella, M. Picardo, T. Sarna, J. D. Simon and S. Ito, Pigm. Cell Melanoma Res., 2013, 26, 616-633.

31 L. L. Chaikov, M. N. Kirichenko, S. V. Krivokhizha and A. R. Zaritskiy, J. Biomed. Opt., 2015, 20, 057003.

32 B. Okutucu, A. Dinçer, Ö. Habib and F. Zıhnıoglu, J. Biochem. Biophys. Methods, 2007, 70, 709-711.

33 S. A. Centeno and J. Shamir, J. Mol. Struct., 2008, 873, 149-159.

34 M. L. Roldán, S. A. Centeno and A. Rizzo, J. Raman Spectrosc., 2014, 45, 1160-1171.

35 M. L. Alfieri, R. Micillo, L. Panzella, O. Crescenzi, S. L. Oscurato, P. Maddalena, A. Napolitano, V. Ball and M. D'Ischia, ACS Appl. Mater. Interfaces, 2018, 10, 7670-7680.

36 R. Micillo, L. Panzella, M. Iacomino, G. Prampolini, I. Cacelli, A. Ferretti, O. Crescenzi, K. Koike, A. Napolitano and M. D’Ischia, Sci. Rep., 2017, 7, 41532.

37 C.-T. Chen, C. Chuang, J. Cao, V. Ball, D. Ruch and M. J. Buehler, Nat. Commun., 2014, 5, 3859.

38 K.-Y. Ju, M. C. Fischer and W. S. Warren, ACS Nano, 2018, 12, 12050-12061.

39 J. R. Hoidal, Am. J. Respir. Cell Mol. Biol., 2001, 25, 661-663. 40 F. Antunes and E. Cadenas, Free Radicals Biol. Med., 2001, 30, 1008-1018.

41 M. Giorgio, M. Trinei, E. Migliaccio and P. G. Pelicci, Nat. Rev. Mol. Cell Biol., 2007, 8, 722-728.

42 J. M. Menter, A. M. Patta, T. D. Hollins, C. L. Moore and I. Willis, Photochem. Photobiol., 1998, 68, 532-537.
43 R. A. W. Smith, B. Garrett, K. R. Naqvi, A. Fülöp, S. P. Godfrey, J. M. Marsh and V. Chechik, Free Radicals Biol. Med., 2017, 108, 110-117.

44 M. Magarelli, P. Passamonti and R. Carlo, Rev. CES Med. Vet. Zootec., 2010, 5, 18-28.

45 P. J. Murray, Annu. Rev. Physiol., 2017, 79, 541-566.

46 P. J. Murray, J. E. Allen, S. K. Biswas, E. A. Fisher, D. W. Gilroy, S. Goerdt, S. Gordon, J. A. Hamilton, L. B. Ivashkiv, T. Lawrence, M. Locati, A. Mantovani, F. O. Martinez, J.-L. Mege, D. M. Mosser, G. Natoli, J. P. Saeij, J. L. Schultze, K. A. Shirey, A. Sica, J. Suttles, I. Udalova, J. A. van Ginderachter, S. N. Vogel and T. A. Wynn, Immunity, 2014, 41, 14-20.

47 P. J. Murray and T. A. Wynn, Nat. Rev. Immunol., 2011, 11, 723-737.

48 T. A. Wynn, A. Chawla and J. W. Pollard, Nature, 2013, 496, 445-455.

49 A. Ortega-Gómez, M. Perretti and O. Soehnlein, EMBO Mol. Med., 2013, 5, 661-674.

50 D. M. Mosser and J. P. Edwards, Nat. Rev. Immunol., 2008, 8, 958-969.

51 K. L. Spiller and T. J. Koh, Adv. Drug Delivery Rev., 2017, 122, 74-83.

52 E. J. Bland, T. Keshavarz and C. Bucke, Mol. Biotechnol., 2001, 19, 125-132.

53 A. Meister and M. E. Anderson, Annu. Rev. Biochem., 1983, 52, 711-760.

54 L. Jin, F. Yuan, C. Chen, J. Wu, R. Gong, G. Yuan, H. Zeng, J. Pei and T. Chen, Inflammation, 2019, 42, 658-671. 